\title{
Practical Use of Oral Rehydration Solutions and their Implementation in ERAS Protocols (Literature Review)
}

\author{
Bihniak PI ${ }^{*}$, Gomon $\mathrm{ML}^{2}$ and $\mathrm{Krenov} \mathrm{K} \mathrm{Yu}^{3}$ \\ ${ }^{1}$ Municipal Non-Commercial Enterprise, "Starokostiantyniv Central District \\ Hospital", Ukraine \\ ${ }^{2}$ National Pirogov Memorial Medical University, Ukraine \\ ${ }^{3}$ Municipal Non-Commercial Enterprise, "Khmelnytskyi Regional Hospital”, Ukraine \\ *Corresponding Author: Bihniak PI, Municipal Non-Commercial Enterprise, \\ "Starokostiantyniv Central District Hospital”, Ukraine.
}

Received: August 09, 2021

Published: September 23, 2021

(C) All rights are reserved by Bihniak PI., et al.

\begin{abstract}
Introduction: Volemic therapy has been paid much attention in the ERAS program, in particular the use of fluids before surgery has been liberalized, the effectiveness of chewing gum has been proved, the early removal of drains and probes has been recommended (which facilitates the intake of fluids and food through the mouth in the postoperative period). The most common electrolyte disorders in the postoperative period is sodium and fluid retention (due to the fact that the operative stress stimulates secretion of antidiuretic hormone), hypokalemia (exchange for hydrogen ions during acidosis compensation, insufficient admission, "invisible losses" in hemolysis, polyuria, diabetes insipidus syndrome, for instance in neurosurgery or sub-compensation of existing diabetes mellitus. The aim of the study was to reveal the influence of preoperative rehydration therapy on patients' functional indicators in early postoperative period.
\end{abstract}

Methods: 73 elective patients of the surgical department of Starokostiantyniv Central District Hospital were examined. The first group consisted of patients receiving "traditional" preoperative preparation (control group) $n=33$. Patients of the second group received IONICA oral rehydration mixture as a preoperative preparation in the amount of $20 \mathrm{ml} / \mathrm{kg}$ the day before surgery, $\mathrm{n}=40$.

Results: The first group $(n=33)$ consisted of patients with "traditional" preoperative preparation, the average age of patients was $43.2 \pm 18.3$, the second group consisted of patients with preoperative preparation, which included the ORM (oral rehydration mixture) IONICA ( $n=40)$, the average age of patients was $46.3 \pm 11.4$. Functional and laboratory indicators were defined before surgery and in 24 hours, and the day of peristalsis recovery was also determined. There was no significant difference between the studied indicators on the first day; the only exception was the glucose level, which was significantly lower in the ORM group in 24 hours compared to the "standard" therapy group. Besides, despite the absence of significant differences in the peristalsis recovery in the control group, it occurred on $2.9 \pm 0.7$, compared with the ORM group: $2.7 \pm 0.6$ day.

Conclusion: ORT is a simple, affordable and effective method to correct hydro-electrolyte balance disorders in elective surgical patients.

Keywords: Oral Rehydration Mixture; Oral Rehydration Therapy

Citation: Bihniak PI., et al. "Practical Use of Oral Rehydration Solutions and their Implementation in ERAS Protocols (Literature Review)". Acta Scientific Gastrointestinal Disorders 4.10 (2021): 64-69. 


\section{Abbreviations}

ORT: Oral Rehydration Therapy; ORM: Oral Rehydration Mixture

\section{Introduction}

The initial use of oral rehydration mixtures (ORM) has proven to be a means to restore circulating blood volume and interstitial fluid in pediatric patients with acute diarrhea. In particular, in 2008, the European Society for Paediatric Gastroenterology, Hepatology and Nutrition (ESPGHAN) and the European Society for Paediatric Infectious Diseases (ESPID) developed recommendations for management of children with acute gastroenteritis. In 2014, the recommendations were revised. In particular, this section brings light to the issue of enteral and parenteral rehydration, correction of hydro-electrolyte balance, complications and monitoring of the disease course. The effectiveness of solutions recommended by ESPGHAN has been demonstrated in several randomized clinical studies and many non-randomized clinical studies. The use of ORM with reduced osmolarity leads to a decrease in the incidence of clinical manifestations (vomiting, defecation frequency, etc.) and reduces the need for additional intravenous therapy (I, A) [1]. According to these recommendations, for children with acute gastroenterocolitis ORM with reduced osmolarity as a first line therapy should be used (I, A). Volemic therapy in the ERAS program has been paid much attention, including liberalized use of fluids before surgery, the proven effectiveness of chewing gum, recommended early removal of drains and probes (which facilitates the intake of fluids and food through the mouth in the postoperative period) [2]. In fact, hypovolemia is a manifestation of acute surgical pathology in most urgent surgical interventions. They, in particular, are accompanied by the loss of circulating blood volume, fluids of the interstitial space (hemorrhage, hypovolemic traumatic shock, mesenteric thrombosis), electrolyte disorders (stenosis of the stomach exit, intestinal obstruction, peritonitis, destructive pancreatitis), accompanying circulatory embarrassments and microcirculations in the intestinal wall. In selective surgery, such abnormalities develop slowly and patients remain compensated for a long time, the microcirculation of the intestinal wall suffers little, hence even mild dehydration (due to hyperthermia, increased diuresis, "traditional" dietary restrictions) can be compensated by means of fluid intake per os [3]. The most common electrolyte disorders in the postoperative period are sodium and fluid retention (due to the fact that the operative stress stimulates the secretion of antidiuretic hor- mone), hypokalemia (exchange for hydrogen ions during acidosis compensation, insufficient admission, "invisible losses" in hemolysis, polyuria, diabetes insipidus syndrome, for instance in neurosurgery or sub-compensation of existing diabetes mellitus. Electrolytes may also be lost during the formation of fistulous passages or increased discharge during draining surgery, complications [4]. Hydro-electrolyte balance correction is a mandatory component of perioperative intensive therapy of surgical patients and is a part of the ERAS European Protocols (Enhanced Recovery After Surgery), where the preoperative volemic preparation and reduction of fasting terms are essential. Peristalsis recovery prevents stasis and bacterial translocation, as a consequence - decrease in the frequency of multiple organ failure in surgical patients [7]. The water content accounts for about $60 \%$ in the adult body. This content varies depending on the age and the size of adipose tissue. The distribution of water is regulated by hydrostatic and osmotic pressure. An integral part of water exchange is the exchange of electrolytes, which creates a stable homeostasis of the internal environment with the ability to auto-regulate upon significant fluctuations in environmental conditions, toxic or viral and bacterial influences. The regulation of water and mineral metabolism is provided by the CNS (antidiuretic hormone of the hypothalamus, deposited in the posterior pituitary and affecting the walls of the distal renal tubules), adrenal glands (aldosterone affects the level of sodium in the blood plasma), kidneys (renin secreted in response to intravascular fluid volume reduction and hypotension), where the processes of filtration and reabsorption take place. The volume constancy and the osmolarity of extracellular fluid is supported by regulatory mechanisms, the main effector organ of which is kidneys. Essential trace elements of the human body are sodium, potassium, calcium, phosphorus, magnesium. The biological effects of these trace elements are bioelectric (development of action potential and rest), osmotic (regulation of osmotic pressure), structural (included in complex proteins and nucleic acids), regulatory (regulation of enzyme activity and transmission of hormonal signal), transport (electron transport with cytochrome iron in the respiratory chain), energy (synthesis of macroergy - ATP, GTP), synthetic (synthesis of hormones, such as iodine in the thyroid hormone), mechanical (included into the bones). Disorders of water and electrolyte exchange are commonly divided into dehydration and hyper hydration. Dewatering (dehydration) develops in cases when the release of water exceeds its flow into the body (negative water balance). Causes can be impaired fluid intake by the organism (water deprivation,

Citation: Bihniak PI., et al. "Practical Use of Oral Rehydration Solutions and their Implementation in ERAS Protocols (Literature Review)". Acta Scientific Gastrointestinal Disorders 4.10 (2021): 64-69. 
coma, esophageal atresia) or increased fluid loss (diarrhea, vomiting, sweating, hyperventilation, polyuria, etc.) [5,6]. The dehydration is divided into iso-, hypo-, hyperosmolar. Isomolar dehydration develops in case of equivalent losses of water and electrolytes. It is observed in acute blood loss, cholera, burns. It is characterized by a decrease in the volume of extracellular fluid without changes in its osmolarity. Laboratory indicators are characterized by an increase in hematocrit, the content of electrolytes is not significantly changed or there is a mild hypokalemia. Hypo-osmolar dehydration develops mainly in case of salts loss, primarily due to the loss of secretions of the stomach and intestines (diarrhea, vomiting), in Addison's disease. Reduction of osmotic pressure in the extracellular space leads to the transition of fluid into cells upon a concentration gradient, resulting in stronger enhancement of the manifestations of dehydration, hemoconcentration and hemodynamic disorders [1-3]. Such type of dehydration quickly leads to changes in the acid-base state manifested by hypochloremic alkalosis (primarily losses of gastric juice containing chlorine) or excretive acidosis (loss of pancreatic or intestinal juice containing sodium and hydrocarbonates). Hyperosmolar dehydration develops in cases when water losses exceed electrolytes losses (primarily sodium). It occurs mainly in case of hyperventilation, fever, increased sweating and reduced water consumption. Oral rehydration therapy as an infectious diarrhea treatment was recommended by WHO in 1979 $[11,12]$. According to WHO estimates, the use of this method in the diarrhea treatment enabled, in particular, to reduce child mortality from 4.6 million in 1980 to 1.5 million in 2000. Initially, first-generation solutions with higher osmolarity level (331 m0sm/l) than blood plasma (285 - $295 \mathrm{mOsm} / \mathrm{l}$ ) have been recommended for the diarrhea treatment, though effectively stop dehydration manifestations and metabolic acidosis. Instead, in 2014, new recommendations were published to substantiate the use of hypo-osmolar solutions (up to $245 \mathrm{mOsm} / \mathrm{l}$ ) with lower concentration of sodium chloride (up to $75 \mathrm{mmol} / \mathrm{l}$ ) and glucose (up to $75 \mathrm{mmol} / \mathrm{l}$ ) [11]. In accordance with the WHO recommendations, the optimal composition of the oral rehydration solution is as follows: sodium - 60 $\mathrm{mmol} / \mathrm{l}$, potassium - $20 \mathrm{mmol} / \mathrm{l}$, bicarbonate (citrate) - $10 \mathrm{mmol} / \mathrm{l}$, glucose - $110 \mathrm{mmol} / \mathrm{l}$. The recommended osmolarity should be close to that existing in the intestinal lumen - $250 \mathrm{mOsmol} / \mathrm{l}$. In all cases in the dehydration treatment, oral fluid administration should be given preference. According to WHO data, when using oral rehydration solutions with balanced composition, the need for intravenous therapy is reduced by $33 \%$ and vomiting frequency by $30 \%$. Oral rehydration is carried out in two stages: stage I - rehydration to eliminate existing intoxication, dehydration and salts loss. Its duration is usually 4 - 6 hours and in $80-84 \%$ of cases of this stage is sufficient to obtain a stable clinical effect $[8,9]$. Stage II - in some patients lasts up to 48 - 72 hours and is a rehydration to eliminate the ongoing losses as well as the intoxication syndrome, the frequency accounts for $14-18 \%$ on the second and $2 \%$ on the third day. The amount of oral rehydration is defined by the dehydration degree and body weight of the patient $[9,10]$. Four dehydration degrees are defined: the first is fluid loss up to $3 \%$ of body weight, the second is loss of $4-6 \%$, the third is loss of $7-9 \%$, and the fourth is uncompensated dehydration (losses exceed $10 \%$ of body weight). Usually, in patients with food toxicoinfections of moderate severity with dehydration of II-III degrees, oral rehydration solutions are used in volume of $40-70 \mathrm{ml} / \mathrm{kg}$. In patients with severe intoxication, but in the absence of dehydration - in the volume of $30-40 \mathrm{ml} / \mathrm{kg}$. In patients with severe intoxication, but in the absence of dehydration - in the volume of $30-40 \mathrm{ml} / \mathrm{kg}$. As already mentioned, oral rehydration mixtures are divided into several generations [11]: I generation - the concentrated WHO mixture (311 mOsm/l), unstable and used until 2004: $3.5 \mathrm{~g}$ of sodium chloride per liter of water, $1.5 \mathrm{~g}$ of potassium chloride, $2.5 \mathrm{~g}$ of baking soda, $20.0 \mathrm{~g}$ of glucose. In case of water-deficient exsiccosis it can enhance hypernatremia. II generation - solutions with an osmolarity of $260 \mathrm{mOsm} / \mathrm{l}$, in which sodium citrate is present instead of soda, which is unstable in solutions, which, with the participation of liver enzymes, is modified into soda. III generation - mixtures with reduced osmolarity (200 - $250 \mathrm{mOsm} / \mathrm{l}$ ), their composition meets the need for the treatment of isotonic, water-deficient, hyponatremic dehydrations. Generation IV - solutions with additional substances, amino acids, etc. to improve absorption [11,12]. IV generation - solutions with additional substances, amino acids, etc. to improve absorption $[11,12]$.

\section{Materials and Methods}

73 elective patients of the surgical department of Starokostiantyniv Central District Hospital were examined. The first group consisted of 33 patients receiving "traditional" preoperative training (control group). It included suspension of nourishment in the evening of the previous day, preparation of the bowel. The state of electrolyte exchange disorders (potassium, sodium, chloride, glu- 
cose level) was evaluated in a laboratory manner on the basis of INVIVO laboratory (Maliks MED LLC). Diuresis indicators, the infusion therapy volume on the first postoperative day, alongside with the duration of intestinal peristalsis recovery were also evaluated. Patients of the second group, 40 people, received IONICA oral rehydration mixture as a preoperative preparation in the amount of $1500 \mathrm{ml}$ the day before surgery.

\section{Results}

The first group ( $\mathrm{n}=33$ ) consisted of patients with "standard" preoperative preparation, men -12 , women - 21 , the average age of patients was $43,2 \pm 18,3$, the second group was patients with preoperative preparation, which included IONICA ORM $(n=40)$, men - 9, women - 31, average age of patients was $46,3 \pm 11,4$. Functional and laboratory indicators were defined before surgery and after 24 hours, and the peristalsis recovery day was also defined. The examination data are summarized in table 1.

There was no significant difference between the studied indicators on the first day; the only exception was the glucose level, which was significantly lower in the ORM group in 24 hours in comparison with the "standard" therapy group. Besides, despite

\begin{tabular}{|c|c|c|c|}
\hline Showing & $n=33$ & $n=40$ & $\mathbf{p}$ \\
\hline Age & $43,2 \pm 18,3$ & $46,3 \pm 11,4$ & $\geq 0,05$ \\
\hline $\begin{array}{l}\text { Infusion volume } \\
\text { per } 24 \text { hours }\end{array}$ & $2627,6 \pm 683,4$ & $2640 \pm 674$ & $\geq 0,05$ \\
\hline $\begin{array}{l}\text { Diuresis on the } \\
\text { first day }\end{array}$ & $1435 \pm 540,4$ & $1370 \pm 282,1$ & $\geq 0,05$ \\
\hline Sodium 0 hours & $135,5 \pm 5$ & $130 \pm 4,9$ & $\geq 0,05$ \\
\hline Sodium 24 hours & $136,9 \pm 6,8$ & $129 \pm 6,5$ & $\geq 0,05$ \\
\hline $\begin{array}{l}\text { Potassium } 0 \\
\text { hours }\end{array}$ & $4,6 \pm 0,8$ & $4,1 \pm 0,9$ & $\geq 0,05$ \\
\hline $\begin{array}{l}\text { Potassium } 24 \\
\text { hours }\end{array}$ & $4,1 \pm 0,8$ & $4 \pm 0,9$ & $\geq 0,05$ \\
\hline Chloride 0 hours & $95 \pm 4,6$ & $93,7 \pm 4,4$ & $\geq 0,05$ \\
\hline $\begin{array}{l}\text { Chloride } 24 \\
\text { hours }\end{array}$ & $95,2 \pm 6$ & $94,5 \pm 4,6$ & $\geq 0,05$ \\
\hline Glucose 0 hours & $5,2 \pm 1,1$ & $4,8 \pm 1,2$ & $\geq 0,05$ \\
\hline Glucose 24 hours & $4,6 \pm 1,3$ & $4,9 \pm 1,2$ & $\leq 0,05$ \\
\hline Peristalsis (day) & $2,9 \pm 0,7$ & $2,7 \pm 0,6$ & $\geq 0,05$ \\
\hline
\end{tabular}

Table 1 the absence of significant differences in the peristalsis recovery in the control group, it occurred on $2.9 \pm 0.7$, compared with the ORM group: $2.7 \pm 0.6$ day.

\section{Discussion}

The traditional use of high volumes of intravenous fluids has the negative effects: polycompartment syndrome, myocardial dysfunction, ARDS syndrome, etc. In particular, data from the Silesian Center for Heart Diseases for 2012 - 2016 indicate that a positive fluid balance of more than $1000 \mathrm{ml}$ in the first 72 hours after admission to the Department of Anesthesiology and Intensive Therapy is independently associated with a lethal outcome in critically ill patients with cardiovascular diseases. It has also been stated that the use of colloids is associated with a higher risk of positive balance [5]. Traditionally, intensive care professionals understand the rehydration therapy as such requiring massive intravenous infusions and using correcting molar solutions (Fesenko, Likhvantsev), even when it is not needed. Stereotypical watching of patients, even those who in the preoperative period have no signs of dehydration and dyselectrolytic manifestations facilitates irrelevant invasions and the development of undesirable effects of hyperhydration, such as swelling of the bowel wall with its subsequent ischemia, intraoperative eruption of ligatures, ischemia of anastomoses and their dehiscence in the postoperative period. Bowel wall edema also contributes to microbial translocation and occurrence of septic complications. Data on the existence of glycocalyx lining the endothelium and its functioning have changed approaches to infusions toward reducing them and even complete discontinuing them in most cases of preoperative preparation. Thus, Fesenko, 2014 indicates changes in the water balance due to a non-specific metabolic response to stress, specific dysfunction of the organ or organ systems, due to the drugs action or limitation of fluid intake, starvation and considers indications for the prescription of infusions Rule 4R: resuscitation - resuscitation, routine maintenance - support by fluid, if the patient is not able to compensate himself independently, replacement - recovery of pathological losses, redistribution - the presence of problems with internal redistribution of fluids [6]. The British Union of Anesthesiologists points to the need for a daily reestimation of the patient's hydrobalance - reassessment [7]. The quintessence of the infusion therapy seminar in Salzburg in 2014 was the conclusion that the optimization of cardiac output is the only indication for infusion therapy (in most cases relative), and the prescription of any intravenous infusion solutions triggers en-

Citation: Bihniak PI., et al. "Practical Use of Oral Rehydration Solutions and their Implementation in ERAS Protocols (Literature Review)". Acta Scientific Gastrointestinal Disorders 4.10 (2021): 64-69. 
dothelial activation and the release of proinflammatory cytokines. Infusion therapy also disrupts the composition of a non-stable glycocalyx by enhancing the secretion of atrial natriuretic peptide. The particular danger is the use of $0.9 \%$ sodium chloride. The tendency for the restrictive infusion therapy is further supported by the fact that the volume overload contributes to the increase of intra-abdominal pressure, with the further enhancement of the intestinal wall microcirculation disorder and again microbial translocation. In this sense, the interest of intensive care professionals to "alternative" ways of fluids administration, which are in fact absolutely physiological, is understandable [8,9]. In general, water and electrolytes are better digested in the intestine from hypoosmolar solutions than from WHO solutions, the need for transition to infusion therapy is less frequent, diarrhea and vomiting were less pronounced, no significant hyponatremia cases occurred [10]. Although the ERAS program has been in use for about 10 years, no data on the type of fluid that would indicate the use of oral rehydration solutions in scientometrical bases has been found. The impact of targeted fluid therapy on enhanced postoperative recovery was studied in 2018 by Hideki Taniguchi., et al. They also put forward a hypothesis that the therapy using oral rehydration solution may be effective for preoperative treatment in surgical patients, before starting the general anesthesia, and studied the safety and efficiency of oral rehydration therapy compared with the intravenous therapy [11]. After treatment, the sodium concentration in the blood and hematocrit values, which decreased within the normal range, were significantly higher in the oral group than in the intravenous group, no significant difference in indicators of serum glucose was observed, the urine volume was significantly higher in the oral group, than in the intravenous group. Patients' satisfaction with the therapy facilitated the ORM therapy based on such factors as "hunger", "dry mouth" and "less exercise limitations." Neither adverse events nor side effects were observed in any of the groups. The results showed that the pre-operative ORM therapy is better than the current pre-operative intravenous therapy in providing with water, electrolytes and carbohydrates, and this therapy should be considered as an alternative to intravenous therapy with fluids and electrolyte control in selected surgical patients who have no reasons to be suspected in a delay in gastric emptying. Similar data was obtained by Hideki Taniguchi., et al. In a pilot study, 20 surgical patients consumed $1000 \mathrm{ml}$ of oral rehydration solution (ORM) 2 hours before starting the general anesthesia. Following parameters such as serum electrolyte concentration, fractional excretion of so- dium (FENa), as an indicator of renal blood flow, esophageal and gastric fluid (EPGF) volume, and patient's satisfaction with ORT were evaluated. In a further study to evaluate ORT safety, 1078 patients with surgery, who consumed ORM 2 hours before the induction of general anesthesia, were evaluated. Results: In a pilot study, water, electrolytes and carbohydrates were efficiently and safely delivered by ORT. These results reveal that ORT is a safe and effective therapy for preoperative treatment of fluid and electrolytes for certain surgical patients [12-14].

\section{Conclusions}

1. There was no significant difference between the studied indicators on the first day; the only exception was the glucose level, which was significantly lower in the ORM group in 24 hours compared to the "standard" therapy group.

2. Despite the absence of significant differences, the peristalsis recovery in the control group occurred earlier: $2.9 \pm 0.7$ days, compared to the ORM group: $2.7 \pm 0.6$ day.

3. ORT is a simple, affordable and effective method to correct hydro-electrolyte balance disorders in elective surgical patients.

\section{Bibliography}

1. European protocol for management of acute gastroenteritis in children (2018).

2. Horelov AV., et al. "Treatment of acute intestinal infections in children: Manual for doctors. - M (2002): 48.

3. Zhydkov YuB and Kolotilov LV. "Infusion-transfusion therapy for infectious diseases in children and adults". M.: MED pressinform (2005).

4. Zakharenko SM. "Oral rehydration therapy for acute intestinal infections”. Journal of Infectology 1.2-3 (2009): 5863.

5. RS Korytniuk., et al. "The role of polyionic solutions in infusion therapy (review)". Pharmatsevt 4 (2012): 37-42.

6. MV Pohorielov., et al. "Modern ideas about water-salt exchange (literature review and methods of own research) Newsletter". Bulletin of Problems Biology and Medicine 2 (2009): 8-14. 
7. Chernii VI and Yevsieieva VV. "Prospects for the application of ERAS protocols in bariatric surgery". Pain, Anesthesia and Intensive Care 82.1 (2018): 29-34.

8. Tatsuya Fukuyama., et al. "Safety and efficacy of oral rehydration therapy until $2 \mathrm{~h}$ before surgery: a multicenter randomized controlled trial Kenji Itou". The Journal of Anesthesia 26 (2012): 20-27.

9. Gustafsson UO., et al. "Guidelines for Perioperative Care in Elective Colonic Surgery: Enhanced Recovery After Surgery (ERAS) Society Recommendations". World Journal of Surgery 37 (2013): 259-284.

10. Toshio Sasaki., et al. "Research Paper Oral Rehydration Therapy for Preoperative Fluid and Electrolyte Management". International Journal of Medical Sciences 8.6 (2011): 501-509.

11. Ewa Trejnowska., et al. "The importance of fluid balance in critically ill patients - a retrospective observational study". Pan African Medical Journal 29 (2018): 208.

12. Pain, anesthesia, intensive therapy. No 3, 2014 Infusion Therapy: Recommendations Based On Common Sense (review of British guideline* for infusion therapy, adapted by U.A. Fesenko) (2014).

13. Hideki Taniguchi., et al. "Cite as Preoperative fluid and electrolyte management with oral rehydration therapy". Journal of Anesthesia 23.2 (2009): 222-229.

14. Hideki Taniguchi., et al. "Cite as Modified ERAS protocol using preoperative oral rehydration therapy: outcomes and issues". Journal of Anesthesia 28.1 (2014): 143-147.

\section{Volume 4 Issue 10 October 2021}

\section{(C) All rights are reserved by Bihniak PI., et al.}

